Investigating the intrinsic cleanliness of automated handling designed for EUV mask pod-in-pod systems

O. Brux, P. van der Walle ${ }^{a}$, J.C.J. van der Donck ${ }^{a}$, P. Dress

SUSS MicroTec Photomask Equipment GmbH \& Co. KG

Ferdinand-von-Steinbeis-Ring 10

75447 Sternenfels

Germany

${ }^{\mathrm{a}} \mathrm{TNO}$

P.O. box 155

2600 AD Delft

The Netherlands 


\title{
Investigating the intrinsic cleanliness of automated handling designed for EUV mask pod-in-pod systems
}

\author{
O. Brux, P. van der Walle ${ }^{\mathrm{a}}$, J.C.J. van der Donck ${ }^{\mathrm{a}}$, P. Dress \\ SUSS MicroTec Photomask Equipment GmbH \& Co. KG, Ferdinand-von-Steinbeis Ring 10, 75447 Sternenfels, \\ Germany \\ ${ }^{\mathrm{a}}$ TNO, P.O. box 155, 2600 AD Delft, The Netherlands
}

\begin{abstract}
Extreme Ultraviolet Lithography (EUVL) is the most promising solution for technology nodes 16nm (hp) and below. However, several unique EUV mask challenges must be resolved for a successful launch of the technology into the market. Uncontrolled introduction of particles and/or contamination into the EUV scanner significantly increases the risk for device yield loss and potentially scanner down-time. With the absence of a pellicle to protect the surface of the EUV mask, a zero particle adder regime between final clean and the point-of-exposure is critical for the active areas of the mask. A Dual Pod concept for handling EUV masks had been proposed by the industry as means to minimize the risk of mask contamination during transport and storage.

SÜSS-HamaTech introduces MaskTrackPro InSync as a fully automated solution for the handling of EUV masks in and out of this Dual Pod System and therefore constitutes an interface between various tools inside the Fab. The intrinsic cleanliness of each individual handling and storage step of the inner shell (EIP) of this Dual Pod and the EUV mask inside the InSync Tool has been investigated to confirm the capability for minimizing the risk of crosscontamination. An Entegris Dual Pod EUV-1000A-A110 has been used for the qualification. The particle detection for the qualification procedure was executed with the TNO’s RapidNano Particle Scanner, qualified for particle sizes down to 50nm (PSL equivalent).

It has been shown that the target specification of $<2$ particles @ 60nm per 25 cycles has been achieved. In case where added particles were measured, the EIP has been identified as a potential root cause for Ni particle generation. Any direct $\mathrm{Ni}-\mathrm{Al}$ contact has to be avoided to mitigate the risk of material abrasion.
\end{abstract}

Keywords: EUV mask, Dual Pod System, EIP, Pod-in-Pod interface, MTPro InSync, intrinsic cleanliness, RapidNano Scanner, Exchange Port, PRP

\section{INTRODUCTION}

The transition from conventional optical lithography to EUVL necessitates significant changes in the system environment and the mask architecture. Whereas 193i lithography is processed under atmospheric conditions using a transmissive reticle, the EUV multi-layer reflective reticle is processed at a shorter wavelength $(13.5 \mathrm{~nm})$ in a vacuum environment. Zero process tolerance levels in respect to backside particle, organic and inorganic contamination require that at-point-of-exposure the integrity of the EUV mask can be guaranteed. Absolute management of the mask and the mask environment is critical to meet this stringent requirement.

Capital investment for a single EUV scanner is nearly $4 \mathrm{x}$ that of a conventional optical lithography scanner. With such a large investment, downtime of the system can not be tolerated and must be avoided at all times. During the Alpha-demo of EUVL at SEMATECH and IMEC, particle cross-contamination between the reticle backside and reticle clamp inside of the scanner not only caused overlay issues but also contributed to significant scanner downtime [1-3]. This heightened the urgency for mask integrity at point-of-exposure. The mask has always important to produce a perfect image on the wafer (yield) but with EUV mask integrity now critical the avoidance of expensive scanner downtime.

The MaskTrackPro (MTPro) advanced mask cleaning platform was developed by SÜSS-HamaTech to specifically address critical mask integrity requirements of next generation lithography including EUVL. Cleaning PORs (Processes of Record) were co-developed on the MTPro to eliminate any negative effects from cleaning on the performance of the reticle. However, the absence of a pellicle leaves the EUV is mask exposed to the environment and particles can be easily deposited onto the mask surface. Prevention of contamination of the mask after final cleaning is critical. A Dual Pod system was developed and introduced into the industry (Figure 1) to reduce the risk of contamination during transportation, handling and storage. 


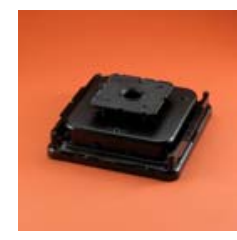

EUV pod assembly. The EUV mask will be stored and transported in the assembly when not in use. Designed for atmospheric and purge storage conditions. Can be handled manually or via automation.

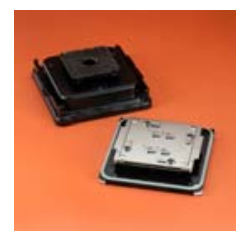

Outer pod cover removed to show EIP. Pod should only be opened by automation in a controlled environment.

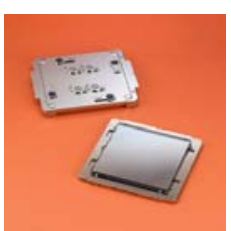

EIP assembly. EIP cover removed to show mask with pattern side down. EIP assembly is placed into exposure chamber. Designed for vacuum use and should only be and led by automation.

Figure 1: Dual Pod System Model EUV-1000. Latest released model EUV-1005 (Courtesy of Entegris Inc.)

The Dual Pod concept presents new challenges [4,5] as the overall infrastructure within the semiconductor fab is not prepared for this type of special handling mechanism. Most photomask equipment can not yet handle reticles in the Dual Pod and must use traditional RSP compatible SMIF boxes. The ability to automate the loading and unloading of the EUVL reticle from the inner pod (EIP) of the Dual Pod and transfer to a cleaning platform is essential for mask integrity requirements. Any manual or semi-manual handling must be eliminated to avoid particle generation. To meet these requirements, SÜSS-HamaTech developed the MaskTrackPro InSync (MTP InSync or InSync) mask management system. The InSync is designed to handle the unique requirements of the Dual Pod and clusters mask cleaning, mask and EIP transfer and storage in a fully-controlled, pristine environment. The MTP InSync module $(2310 x 160 / 250 \mathrm{~cm})$ can be clustered to the MaskTrackPro cleaning platform or used as stand alone system as shown in Figure 2. In either case, the intrinsic purpose of MaskTrackPro is to load/unload the EIP from the outer shell of the Dual Pod and handle the EUVL reticle into/out of the EIP. If clustered with the MaskTrackPro cleaning system the MTP InSync manages the transfer of the reticle to and from the cleaning platform. MTP InSync is capable of handling the reticle into/out of a standard SMIF Pod or inspection tool.
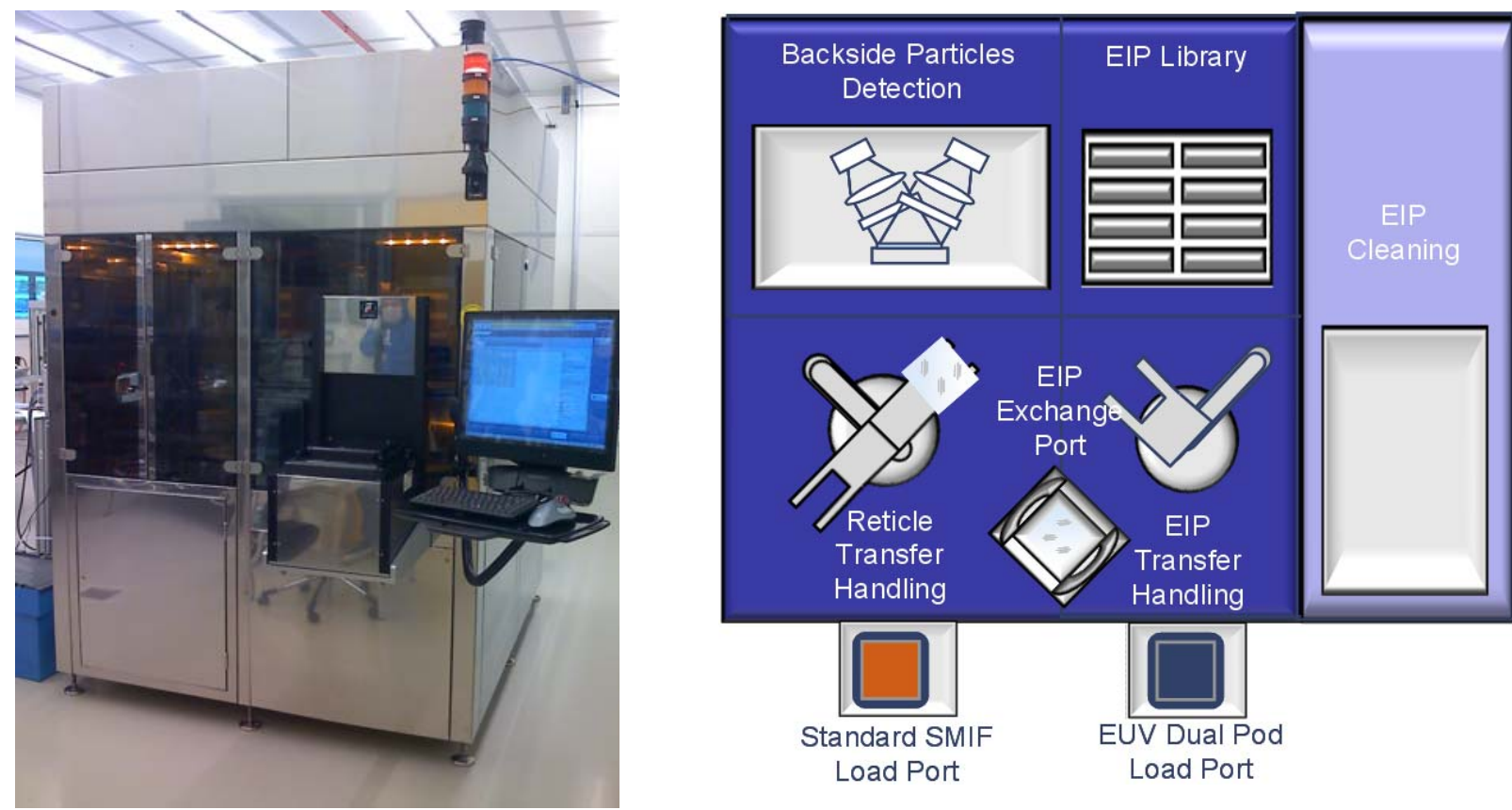

Figure 2: Image of MTP InSync during the qualification period at TNO (left side). On the right, is an illustration of a full equipped MaskTrackPro InSync tool, comprising of a Dual Pod load pod, SMIF pod opener, exchange port, EIP library, and two robots for EIP and EUV reticle handling.

Essential to the EIP automation is a specially designed exchange port integrated into the MTP InSync. The exchange port opens the EIP to make the EUVL reticle accessible. The interfacial exchange port separates two different classes of controlled environments:

1. The EIP transfer handling area where the EUVL reticle is still protected by the EIP.

2. The Reticle handling area where the unprotected EUV reticle is exposed to the environment. This environment can be controlled by XCDA, to avoid any (re) contamination of the reticle with moisture.

To avoid cross-contamination between EIP and reticle, the EIP requires specific cleaning treatments. MTP InSync offers a variety of optional features such as particle removal with a dry cleaning method that is able to access all small voids of in the complex surface structure of the inner shell. For preservation of the inner and outer surface conditions, the EIP is stored in a special library under Nitrogen (N2) purged atmosphere until such time that it is loaded with a 
cleaned reticle. The InSync allows for eight EIPs to be stored at any given time to allow EUV reticle cleaning for high volume manufacturing. Backside particle detection functionality based on a full substrate illumination technology can be integrated into the MTPro InSync. The detection of particles down to 150nm allows for verification of EUV reticle cleaning process and ensures that a contaminated substrate will not be returned into the Dual pod and subsequently into the vacuum environment of the EUV exposure tool. All functional units must avoid and control particle contamination during transport and handling. It is mandatory that path through the MTP InSync itself does not contaminate the EIP and the reticle. An investigation of the intrinsic cleanliness of each MTP InSync component is indispensable.

SÜSS-HamaTech and TNO (Netherlands) have cooperated in an effort to qualify the MTP InSync intrinsic cleanliness. The MTP InSync tool used for the qualification is installed at IMEC. It was designed to be clustered the MTPro cleaning platform and comprised of a Dual Pod load port station, EIP exchange port, an eight slot EIP library and robot handlings for reticle and EIP transfer. Particle generation for these InSync base components that could lead to crosscontamination of the EUV reticle must be minimized.

The following paper describes various pre-defined full cycle and isolation tests run to verify the cleanliness of the MTP InSync and its components.

\section{EXPERIMENTAL}

\subsection{Samples and qualification approach}

To qualify intrinsic cleanliness of the InSync tool, two different samples were used; a "wafer sample” and a EUV mask blank. The "wafer sample" comprised of an aluminium plate in the same physical dimensions and shape as that of a standard EUV mask blank and a 4" silicon wafer mounted to the surface of the plate. The wafer was mounted such that the total thickness of the reticle did not exceed the thickness of a standard EUV mask blank of 0.25”.
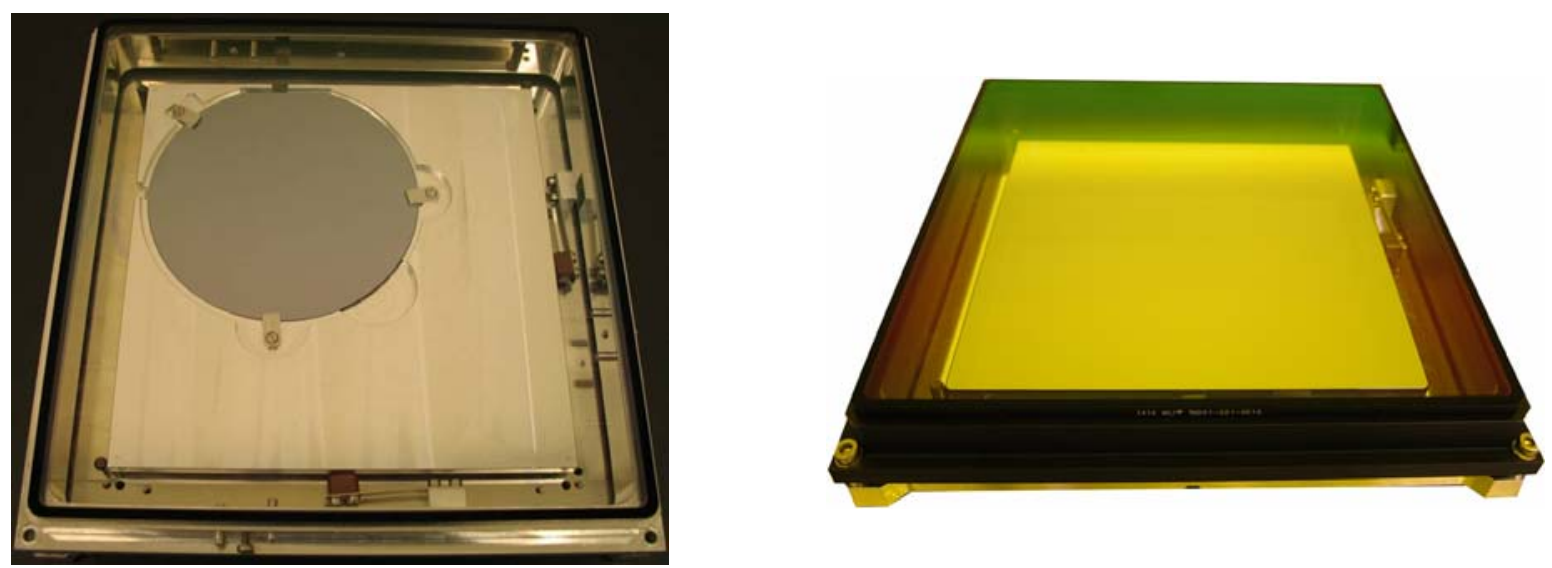

Figure 3: Images of the "wafer sample" and EUV mask blank sample used during the qualification of intrinsic cleanliness. The "wafer sample” comprised of a 6”x6” 0.25 ” aluminium plate and a 4" wafer (left photo). The mask sample used was a standard EUV mask blank (right photo). Both samples are shown in the RapidNano scan-box.

Figure 3 (left photo) shows an image of the "wafer sample" inside the scan-box of the RapidNano [6,7] (defect detection system by TNO, see also chapter 2.2). Wafer level particle analysis was performed on the "wafer sample" using SEM and EDX in those cases where added particles detected were of a larger size. The second sample used in this evaluation was a standard EUV mask blank with a 2.5nm Ru capping layer on the front side and CrN layer on the backside. Figure 3 (right photo) shows an image of the EUV mask blank in the scan-box of the RapidNano. The EUV blank was used to determine the adder level from handling run in the InSync tool. The standard mask form factor of both samples allowed for an automated handling inside the InSync tool and minimized the risk of associated with manual handling.

The intrinsic cleanliness of the MTP InSync tool is characterized by the number of added Particles per Reticle Path (PRP). The added PRP specification value is defined to 0.08 for particles larger 60nm (PSL equivalent) or less than 2 particles added for the 25 total handling paths in the InSync tool (see figure 4, qualification routing \#4). These test cycles are were designed according to the principles of acceptance sampling $[6,8,9]$. In pre-qualification runs, the adder level of the manual transfer of the samples (hand carried in the Dual-Pod System) between the InSync tool and the RapidNano inspection system and the contribution of the Dual Pod and the measurement sequence itself was determined to result in a total adder PRP value of 0.05 (particle size larger 60nm PSL equivalent): This value was considered for the up-coming qualification experiments as the background particle adder level.

Figure 4 provides an overview of the mask routings in the InSync tool that were qualified for contributions to the adder PRP value. The arrows illustrate the sequence of the individual routings and on the right side of Figure 4, a short description of each routing is provided. Routing \#4 represents the complete path of the EUV mask and the appendant 
EIP while all other routings are sub-routings thereof. The total handling path (routing \#4, Figure 4) is comprised of the following automated handling sub-routings:

$\checkmark \quad$ Unload EIP (with the mask) from the outer pod located on the Dual Pod load port (routing \#1, fig. 4)

$\checkmark \quad$ Transfer EIP into the exchange port and open (routing \#5, fig. 4)

$\checkmark \quad$ Unload the mask from the EIP while in the exchange port (routing \#6, fig. 4)

$\checkmark \quad$ Close and transfer empty EIP from the exchange port into a library slot (routing \#5 \& \#2, fig. 4)

$\checkmark \quad$ Transfer empty EIP from the library slot into exchange port and open (routing \#2 \& \#5, fig. 4)

$\checkmark \quad$ Load mask into the EIP while in the exchange port (routing \#6, fig. 4)

$\checkmark \quad$ Close and transfer EIP (with the mask) out of the exchange port (routing \#5, fig. 4)

$\checkmark \quad$ Transfer EIP (with the mask) into the outer pod located on the Dual Pod load port (routing \#1, fig. 4)

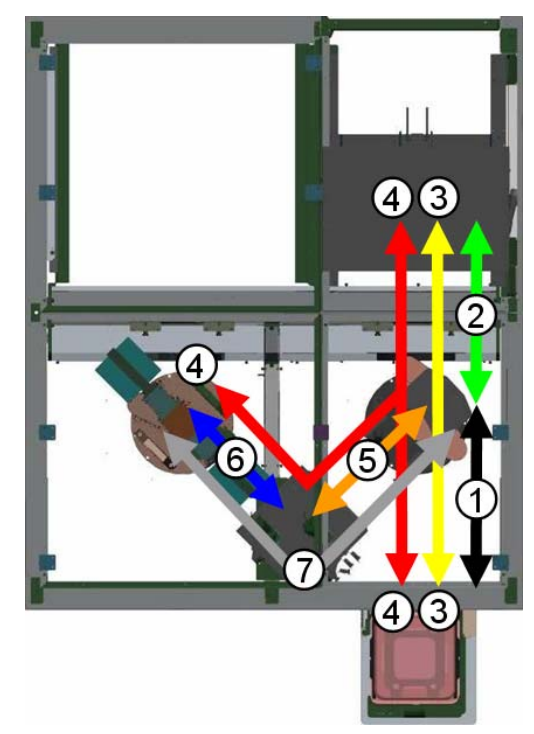

\section{Qualification routings}

1. Unload EIP with the mask from the outer pod on the Dual Port load port and load EIP with the mask into the outer pod on the Dual Pod load port.

2. Load EIP with the mask into several library slots and unload EIP with the mask out of the library slots.

3. Load EIP with the mask from the Dual Pod load port to a library slot and unload EIP with mask from the library slot into the Dual Pod load port.

4. Total handling cycle: Load EIP to the exchange port, unload the mask out of the EIP, load the empty EIP to a library slot, and reverse all movement until the EIP with the mask is loaded back to the outer pod on the Dual Pod load port.

5. Load EIP with the mask to the exchange port including the opening of the EIP and unload EIP with mask from the exchange port

6. Unload the mask from the EIP in the exchange station and load the mask back into the EIP while in the exchange station

7. Combination of routings \#5 and \#6

Figure 4: The sketch illustrates the sequence of the individual routings in the InSync tool.

The total handling cycle (routing \#4, Figure 4) and sub-routings are recipe controlled by InSync software and were run automatically during the qualification period at TNO. Each sequence is performed at least 168 times to provide statistically meaningful information to compare pre- and post defect inspection results (see chapter 2.2 for details). In the tests above only 1 library slot was used. In order to show that these results are valid for all slots, the equivalence between the different library slots was tested. For this test it was assumed that a faulty library slot would have a PRP > 0.1 and that a functioning library slot would have a PRP $=0.01$. Three possible scenarios were distinguished:

1) 7 slots function at a PRP level $=0.01$

2) 6 slots function at a PRP level $=0.01$ and 1 slot function at a PRP level $=0.1$

3) 6 slots function at a PRP level $=0$ level and 1 slot function at a PRP level $=0.1$.

At a minimum, 250 cycles would need to be carried out per slot to distinguish between scenarios 1 \& 2; 7 slots function at a PRP level $=0.01$ versus 6 slots functioning at a PRP level $=0.01$ level with 1 slot functioning at a PRP level $=0.1$.

265 cycles were performed per slot on 7 slots. The expected values for each of the three scenarios were calculated (see Figure 5). From the calculations it was observed that if less than 18 particles were observed none of the slots performed at a higher PRP level than 0.1 . If more than 28 particles were observed a faulty slot is presumed. If the number of added particles falls between 18 and 38, a single slot multi-load test (795 cycles) should be performed. If the number of added particles lies between 3 and 13 and the outcome of the 7 slot cycle test is between 11 and 28 particles, it can be determined that the results agree with an average PRP level $=0.01$ and all slots are equivalent. 


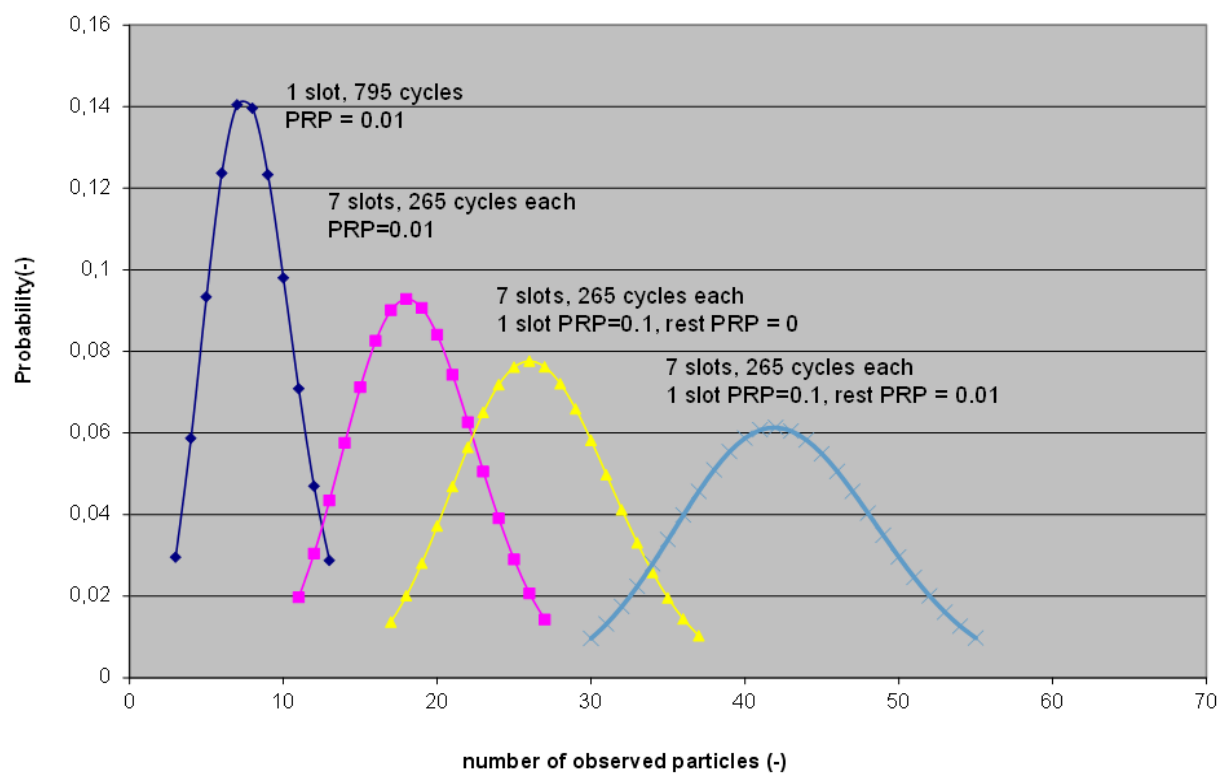

Figure 5: Expected outcomes for different scenarios of the library test

\subsection{Defect detection by RapidNano Particle Scanner}

The RapidNano Particle Scanner is capable of detecting 1 added particle $(>50 \mathrm{~nm})$ on a reticle sized substrate [6,9]. In a step and scan mode, dark field images are acquired of the entire reticle area. The location and size of particles are determined from the images and processed into defect-maps and particle size distributions (see Figure 6). The particle size distribution does not always give an accurate representation of the number of observed particles. Large particles often cause heavy and fragmented scattering whereby the original particle is surrounded by a large cluster of (false) detections. Therefore, the differential of the particle size distributions of the Pre- and Post Defect maps will provide faulty results. A correct adder map can be processed by linking particle locations from Pre- and Post- defect maps to generate a list of "added" and "lost” particles. The lists are checked visually and all fragmented or clustered scatterings are removed, resulting in a list of the true "added” particles.

Pre-defect map $\quad$ Post-defect map Adder- defect map
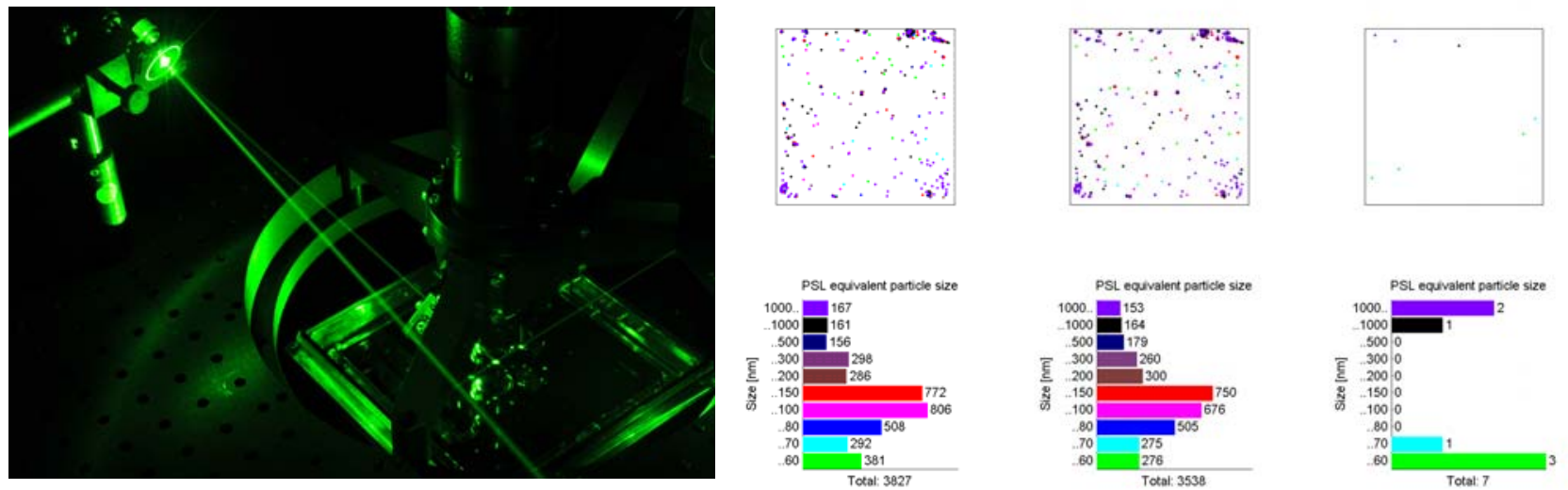

Figure 6: Interior view of the RapidNano Particle Scanner (left photo); Examples of defect diagrams before (Pre), after (Post), and the resulting adder counts for routing \#5 (refer to Figure 4) with 575 repeated cycles. The upper images depict the defect mapping on the inspection area of the mask surface and the lower images show a defect size histogram graph. 


\section{RESULTS AND DISCUSSION}

\subsection{Intrinsic cleanliness qualification results}

Intrinsic cleanliness experiments were performed by measuring the defect map on the EUV blank surface before and after running a number of test cycles with a dedicated routine (refer to Figure 4). During these cycles the EUV mask was orientated with the defect inspected side facing down to mimic the standard mask orientation in an EIP. Table 1 summarizes the results of the experiments by listing the number of repeated cycles, total added particles, and calculated PRP value. Most of the routings showed a PRP value of 0.02 or lower, which is sufficiently small enough to meet the specified PRP value of less than 0.08 for the total process flow in the InSync tool. The $95 \%$ confidence intervals of the internal handling units were all $<0.055$ and meet the requirement of $<2$ particles added per 25 cycles. Routings \#3 and \#4 include transfer handling to/from the external load port to/from any other station. These routings resulted in a high amount of added particles. Particle sizes larger $1 \mu \mathrm{m}$ were found which indicate that the particles were produced from mechanical friction. In a visual check it was noted that the placement of the EIP in the load port was not completely smooth. Improvement of the pick and place procedure by the EIP robot will correct that issue. Further detailed investigations such as defect map signature and material analysis identified possible root causes. Tool related issues have been resolved.

\begin{tabular}{|c|c|c|c|c|}
\hline Routing \# & Number of cycles & Total adder count & PRP & 95 \% interval \\
\hline 1 & 168 & 2 & 0.012 & $0.004-0.043$ \\
\hline 2 & 191 & 4 & 0.021 & $0.008-0.054$ \\
\hline 3 & 184 & 23 & 0.125 & $0.013-0.191$ \\
\hline 4 & 183 & 87 & 0.475 & $0.05-0.59$ \\
\hline 5 & 575 & 7 & 0.012 & $0.006-0.025$ \\
\hline 6 & 641 & 6 & 0.009 & $0.004-0.020$ \\
\hline 7 & 296 & 6 & 0.020 & $0.010-0.044$ \\
\hline
\end{tabular}

Table 1: Overview of the results from individual intrinsic qualification runs by routing \# (refer to Figure 4), number of cycles performed, resulting adder count and calculated averaged particles added per mask cycle (PRP).

The library equivalence was tested by loading and unloading the EIP with a reticle in slot 2 to 8 each for 265 cycles. After a total of 1855 cycles, 1 added particle was observed. From this result it was concluded that the library slots were equivalent.

\subsection{Root cause analysis for adders}

A high number of particles were observed on the pattern side of the sample after a full cycle was performed. A partial cycle in the exchange port revealed an even higher number of particles on the reticle back side. By using the "wafer sample” (see Figure 3) with the wafer facing up and routing \#5, particles were collected in the exchange port. In total 30 particles $(>1 \mu \mathrm{m})$ were added to a $40 \mathrm{~cm}^{2}$ area (Table 2). Seven particles were found in a SEM analysis and the composition of these particles were determined by EDX analysis (see Figure 7).

\begin{tabular}{|l|c|c|c|}
\hline Defect size range & Pre-Defects & Post-Defects & Added Defects \\
\hline$>\mathbf{1 0 0 0} \mathbf{~ n m}$ & 12 & 41 & 30 \\
\hline $\mathbf{5 0 0} \mathbf{n n}-\mathbf{1 0 0 0} \mathbf{~ n m}$ & 9 & 11 & 1 \\
\hline
\end{tabular}

Table 2: Defect counts on wafer for SEM-EDX measurements

Three particles contained nickel (A, B and C; figure 7a). Particles A and B also contained aluminium and magnesium peaks. The silicon peaks originated from the wafer substrate. Since Nickel is only used as plating of the EIP, these Nickel particles were determined to have originated from the EIP. Particles D, E and F (figure 7b) were primarily aluminium however, the composition of trace materials was different for each of the particles. Particle D contained magnesium. This is a common alloy for aluminium and therefore was not unexpected. Particle E had a small shoulder at the magnesium position. However, the magnesium / aluminium ratios of these particles were very different and were determined to have originated from different sources. The magnesium / aluminium ratio of particle D was comparable with the ratios in particles A and B. It is very likely that particle D also originated from the EIP. Other sources, such as points of contact between IP lid and support in the exchange port cannot be excluded. Particle F contained calcium and 


\section{SÜS,MicroTec}

sodium and it is very likely to have come from a third source. A seventh particle was found with a similar composition as particle $\mathrm{E}$.

A
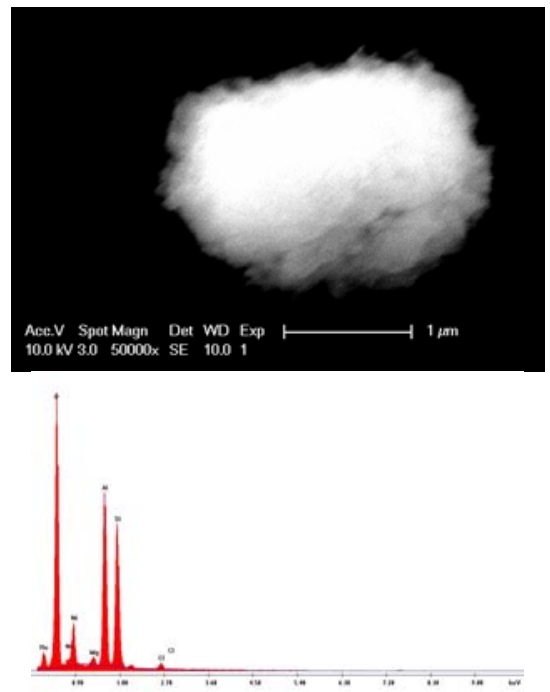

B
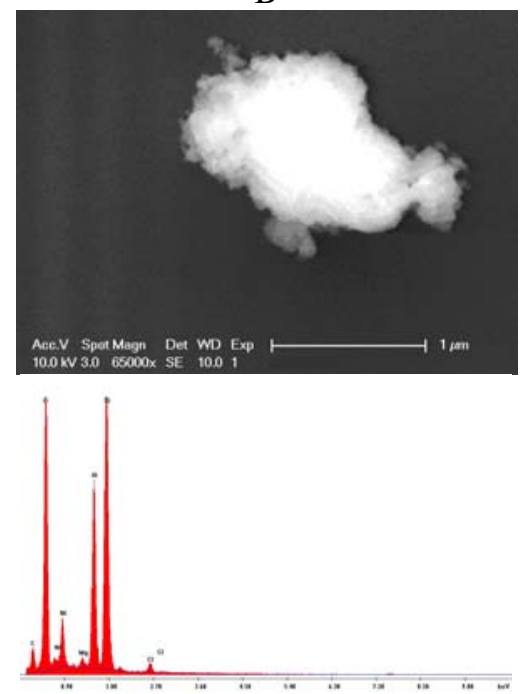

C
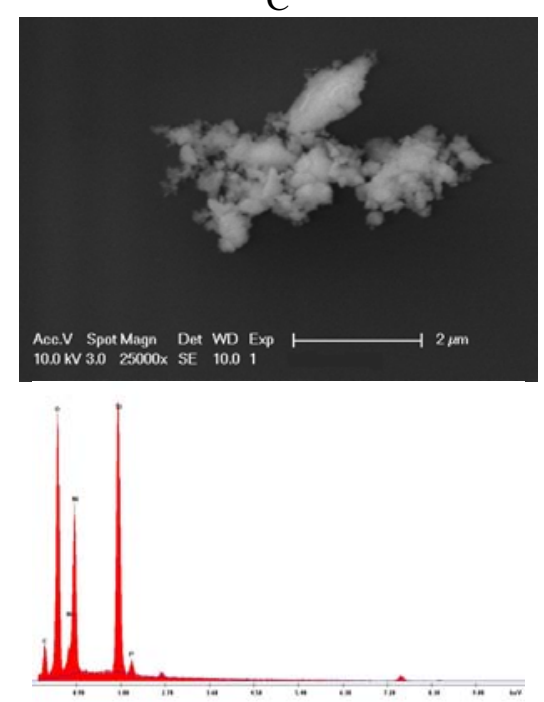

Figure 7a: Images of typical adders found (wafer sample tests). on the upper row are SEM images of added particles, whereas on the lower row show the corresponding EDX spectra of each particle.

$\mathrm{D}$
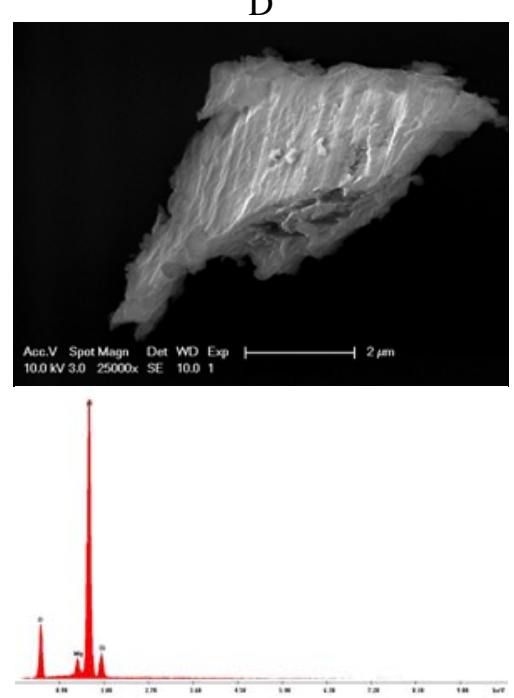

$\mathrm{E}$
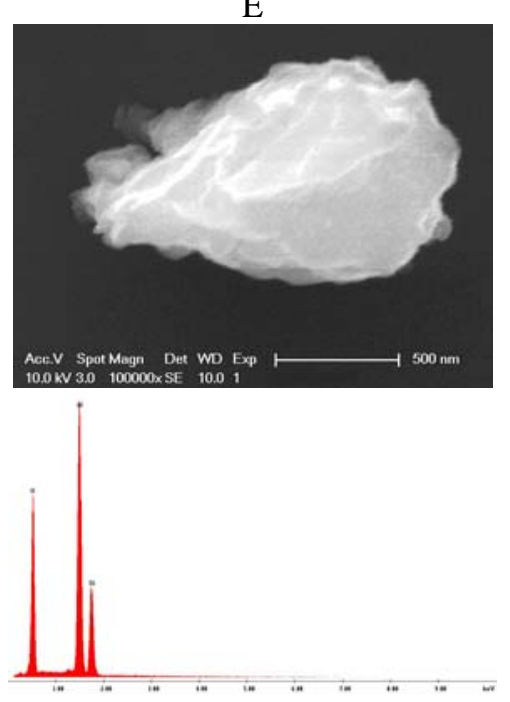

F
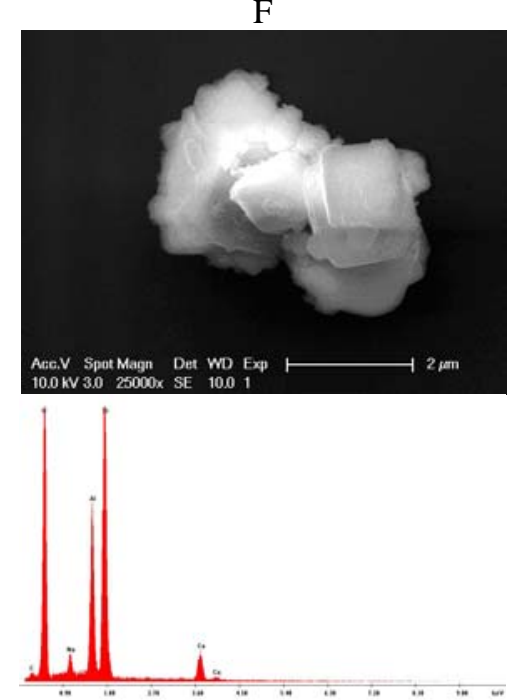

Figure 7b: Images of typical adders found (wafer sample tests). on the upper row are SEM images of added particles, whereas on the lower row show the corresponding EDX spectra of each particle.

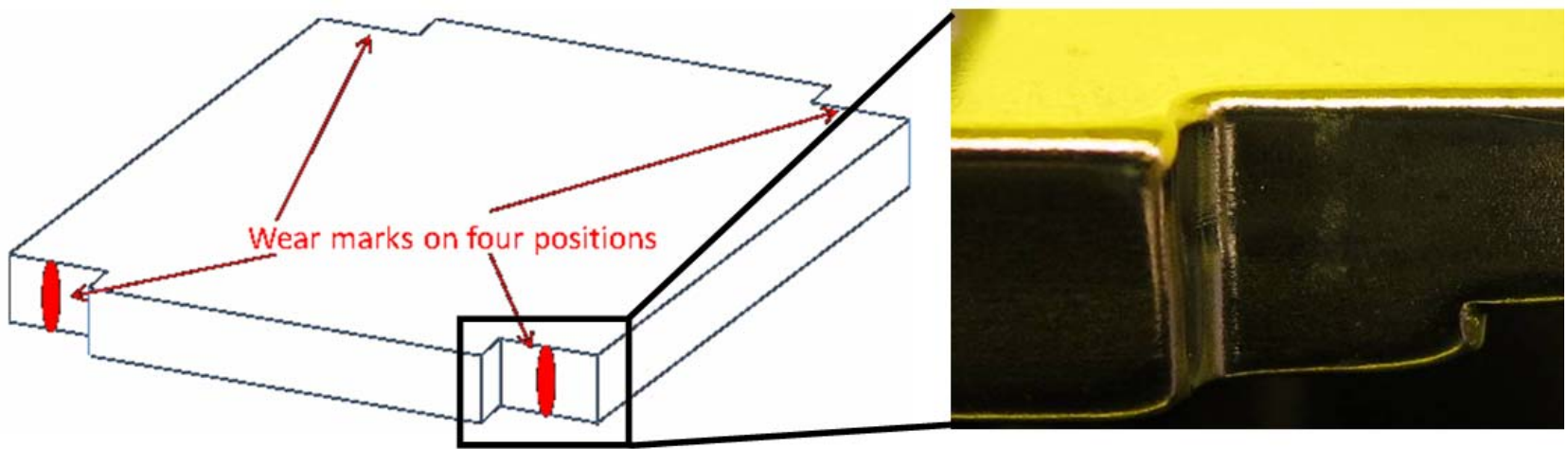


Figure 8: The image on the right shows the location of wear mark on bottom plate of EIP after more than 4000 cycles; the illustration on the left highlights the locations of the material wear seen in the image on the right.

Visual inspection of the EIP revealed wear marks on the side of the bottom plate at a point where the lid touches the bottom plate and can be seen in Figure 8. The illustration in Figure 8 highlights the location of the wear marks. Particles were collected from these wear mark location with a carbon sticker. Analyzed by SEM (see Figure 9; particle SEM image on the left, EDX graph on the right), a large number of particles were present. The EDX spectrum analysis detected three typical components; nickel, aluminium and magnesium. Since carbon was used instead of a silica wafer as the background, the silica peak disappeared. The magnesium / aluminium ratio was comparable to the ratios observed in particles A, B and D in Figure 7. It can be determined that at least 4 of the seven particles were attributable to the EIP. The high aluminium concentration compared to nickel indicates that of the majority of the nickel plating had been removed.
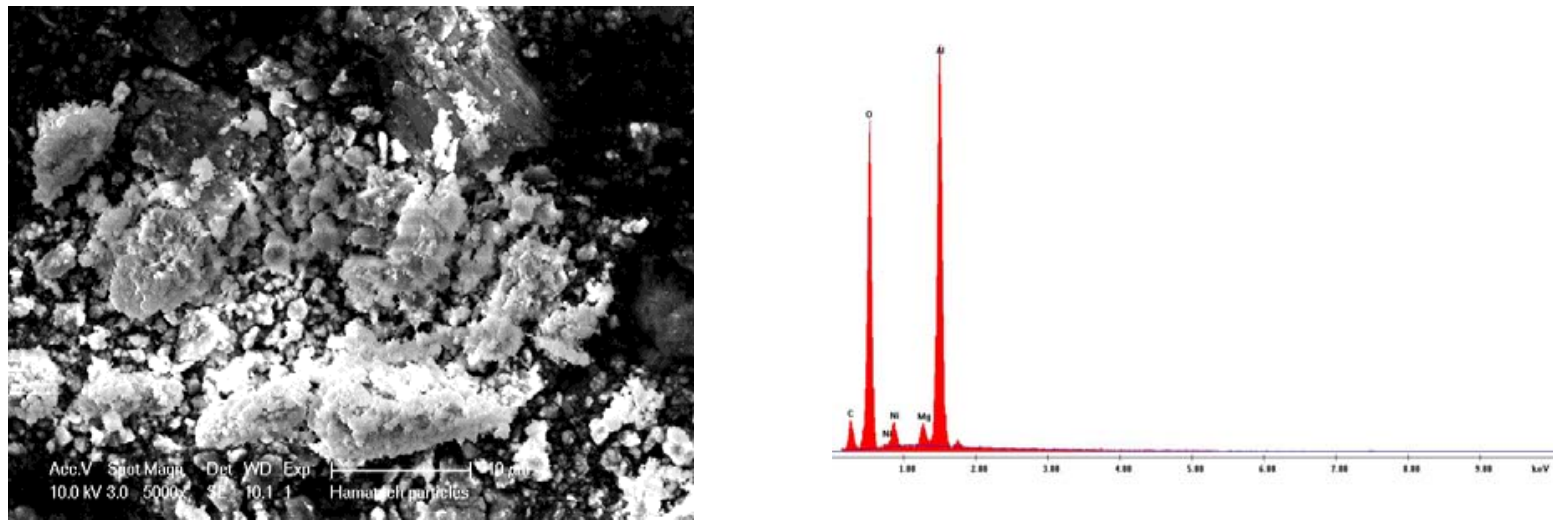

Figure 9: SEM image (left) and EDX spectrum (right) of particles collected at wear marks on the EIP

Before the experiment, 21 particle $(>500 \mathrm{~nm})$ were present on the wafer. During the experiment 31 large particles were added and consequently, only $60 \%$ of these particles were related to the experiment. Three different types of particles (and thus sources) were observed. Four particles could be attributed to the EIP, 2 particles were aluminium only and one particle contained sodium and calcium. The oxygen/aluminium ratio in particles E (aluminium only) was much higher than particle D. This leads to the conclusion that particle D was freshly formed while particles E were much older. One “aluminium only" particle, particle E, was smaller than $1 \mu \mathrm{m}$. As seen in Table 2, $90 \%$ of the particles in the $500-1000$ $\mathrm{nm}$ category were present on the wafer before the experiment. It can be surmised that pure aluminium particles (E) probably originated from a source outside of the InSync tool. 


\section{CONCLUSIONS}

In this study, a Dual Pod System EUV-1000A and a standard EUV blank were used to characterize the intrinsic cleanliness performance of MTP InSync. By a various number of test cycles and isolation tests, potential particle sources have been determined and the material of particles was investigated. It was distinguished that particle free handling and transfer of the EUV reticle is achievable once the accuracy of the pick and place procedure via the EIP robot has been ensured. The Dual Pod Load Port, EIP transfer handling, EIP library and the handling of the EUV blank on the reticle transfer robot showed good results in respect to particle adders. The target specification of $<0.08$ PRP was achieved.

During some full cycle tests, particle generation was observed. Additional isolation tests with consecutive material analysis identified Nickel (Ni) as material. In combination with the observation of wear on the EIP at the contact point between bottom and lid of the EIP, particle generation caused by abrasion of the Ni plated EIP during the opening and closing was another root cause. This abrasion occurred after approx. 4000 openings and closings.

In addition, Aluminium (Al) particles were identified which either originated from the EIP (bulk material of the EIP, Ni plating completely worn out) or from the Ni-Al direct contact of the EIP inside the Exchange Port (Aluminium support for the EIP lid). Based on these experiences, the design of the Exchange Port was improved to avoid direct Ni-Al contact surface. Particle generation caused by friction on the EIP during opening and closing requires further modification of the EIP and must be addressed to the Dual Pod supplier.

\section{ACKNOWLEDGEMENT}

Authors would like to acknowledge Rik Jonckheere (IMEC) for providing the EUV mask blanks, Hans van den Berg from TNO for the preparing the SEM images and EDX spectra during the material analysis.

HamaTech APE gratefully acknowledges the financial support for part of this work by the European community under

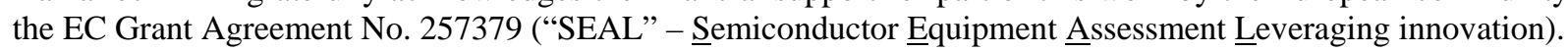

\section{REFERENCES}

1. S. Lee, T. Yamamoto, K. Ota, N. Nishimura, T. Taguchi, I. Nishiyama, O. Suga, S. Warisawa, S. Ishihara "Analysis of entrapped object size effects on Out-of-Plane Distortion of the EUVL mask in electrostatic chucking" Int. EUVL Symposium 29 Sept. - 2 Oct. 2008

2. O. Wood, E. Gallagher, L. Kindt, M. Barrett, H. Kato, U. Okoroanyanwu1, J. Schefske, A. Raghunathan, T. Wallow, J. Whang, C. Boye, S. Kini: „Impact of frequent particle removal on EUV mask lifetime“; Int. EUVL Symposium 19 Oct. 2010

3. A.V. Hayes, R. Randive, I. Reiss, J. Menendez, P. Kearney, T. Sugiyama: "Evaluation of backside particle contamination and electrostatic chuck design on the cleanliness of EUV reticle mask blanks in a multilayer Mo/Si ion beam deposition system“; SPIE Proc. Vol. 7122 71223O-1 (2008)

4. J. Zimmerman, L. He: "Progress on EUV reticle dual pod carriers for use in the fab and exposure tools"; Int. EUVL Symposium, 29 Sept. - 2 Oct. 2008

5. A. Rastegar, A.J. Kadaksham, B. Lee, M. House, D. Ashworth, M. Kishimoto: "Substrate, Blank and Mask Cleaning Contribution to EUV Mask Defectivity and Lifetime“; Int. EUVL Symposium 19 Oct. 2010

6. J.C.J van der Donck, R. Snel, J.K. Stortelder, A. Abutan, S. Oostrom, S. van Reek, B. van der Zwan, P. van der Walle: "Particle detection on flat surfaces"; SPIE Proc. Vol. 7969 (2011)

7. J.K. Stortelder, J.C.J. van der Donck, S. Oostrom, P. van der Walle, O. Brux, P. Dress: "Particle qualification procedure for the TNO EUV reticle load port module of the HamaTech MaskTrackPro cleaning tool”; SPIE Proc. Vol. 7969-60 (2011)

8. J.L. Devore: "Probability and Statistics for Engineering and the Sciences"; (5th edition), Duxbury, Pacific Grove, pp. 709-714 (2000)

9. J.C.J. van der Donck, J.K. Stortelder, G.B. Derksen: “Optimized qualification protocol on particle cleanliness for EUV mask infrastructure“; SPIE Proc. Vol. 8166-96 (2011) 